\title{
Successful Endovascular Management of Common Femoral Artery Perforation During Cannulation for Extracorporeal Membrane Oxygenation
}

\author{
Su Nam Lee, ${ }^{1} \mathrm{MD}$, Ki-Dong Yoo, ${ }^{1} \mathrm{MD}$ and Min Seop Jo, ${ }^{2} \mathrm{MD}$
}

\begin{abstract}
Summary
In massive pulmonary thromboembolism, requiring cardiopulmonary resuscitation, venous-arterial extracorporeal membrane oxygenation can decompress the overloaded right atrium and ventricle while stabilizing hemodynamic status. However, vascular injuries occur in slightly less than $20 \%$ of cases during cannulation. In a $44-$ year-old woman with suspected pulmonary thromboembolism, a common femoral artery perforation occurred unexpectedly during cannulation for extracorporeal membrane oxygenation. The patient was unstable due to active bleeding. Fortunately, we occluded the bleeding site using a stent. Interventional cardiologists are frequently challenged by vascular injury during cannulation for extracorporeal membrane oxygenation. We recommend imaging modalities to prevent vascular complications. However, if vascular injuries occur, stent placement should be considered for patients with arterial perforation during cannulation for extracorporeal membrane oxygenation.
\end{abstract}

(Int Heart J 2019; 60: 231-234)

Key words: Massive pulmonary thromboembolism, Vascular complication, Hemodynamic instability

$\mathrm{M}$ assive pulmonary thromboembolism (PE) is defined as an embolus causing sufficient blockage to pulmonary flow, resulting in hemodynamic instability, hypoxia, and right heart failure. Massive PE is associated with poor clinical outcome. ${ }^{1)}$ Early mortality in patients with massive $\mathrm{PE}$ is at least $15 \%$, and the mortality rate in patients requiring cardiopulmonary resuscitation (CPR) is as high as $64.8 \% .^{2)}$ High-dose unfractionated heparin should be administered upon suspicion of massive PE. Systemic fibrinolysis is the first-line treatment in patients with massive PE, although it is not worth the risk in all patients with acute PE. ${ }^{3)}$ Other treatment methods include open surgical embolectomy and catheter thrombectomy.

Venous-arterial (V-A) extracorporeal membrane oxygenation (ECMO) improves right heart failure, and stabilizes hemodynamic status in critically ill patients with massive PE who are too unstable to tolerate other interventions ${ }^{1)}$. However, vascular complications occur in fewer than $20 \%$ of cases during cannulation for ECMO, but the consequences are serious. ${ }^{4)}$ This negatively affects survival in patients receiving veno-arterial ECMO. ${ }^{4}$ Herein, we report the successful endovascular management of common femoral artery (CFA) perforation during cannulation for $\mathrm{ECMO}$ in a patient requiring $\mathrm{CPR}$ due to massive $\mathrm{PE}$.

\section{Case Report}

A 44-year-old woman with no prior history of disease was referred to the emergency room with severe dyspnea for one week. One month earlier, she had applied a splint to her right ankle due to a sprain. Clinical examination revealed signs of cardiogenic shock, including low blood pressure $(70 / 40 \mathrm{mmHg})$, tachycardia (heart rate 120/minute), and tachypnea (respiratory rate 30/minute). Chest X-rays were normal. An electrocardiogram (ECG) showed sinus tachycardia with S1Q3T3. Laboratory testing revealed an elevated d-dimer level (6.19 mg/L). Transthoracic echocardiography (TTE) revealed a Dshaped left ventricle (Figure 1A), along with right atrium dilation and right ventricle cavities (Figure 1B), pulmonary hypertension (RVSP $56.6 \mathrm{mmHg}$, Figure 1C) and RV dysfunction (TAPSE $8 \mathrm{~mm}$, Figure 1D). Clinically, PE was suspected. The patient arrested ten minutes after her arrival and CPR was started. During CPR, V-A ECMO was applied for hemodynamic stabilization. ECMO insertion was attempted via the left femoral artery and left femoral vein because the intravenous line was only in the right femoral vein. However, the left site approach failed because the patient was obese (body mass index: 28.7), and an acute hematoma developed at her left thigh and abdomen. Manual compression was applied at the hematoma site, and ECMO was applied via the right femoral artery and right femoral vein. The Capiox (Terumo, To-

From the 'Department of Internal Medicine, St. Vincent's Hospital, The Catholic University of Korea, Suwon, South Korea and ${ }^{2}$ Department of Thoracic and Cardiovascular Surgery, St. Vincent's Hospital, The Catholic University of Korea, Suwon, South Korea.

Address for correspondence: Ki-Dong Yoo, MD, Department of Internal Medicine, St. Vincent's Hospital, The Catholic University of Korea, 93, Jungbudaero, Paldal-gu, Suwon-si, Gyeonggi-do, 16247, South Korea. E-mail: cardioyoo@gmail.com

Received for publication April 25, 2018. Revised and accepted June 12, 2018.

Released in advance online on J-STAGE November 5, 2018.

doi: 10.1536/ihj.18-266

All rights reserved by the International Heart Journal Association. 


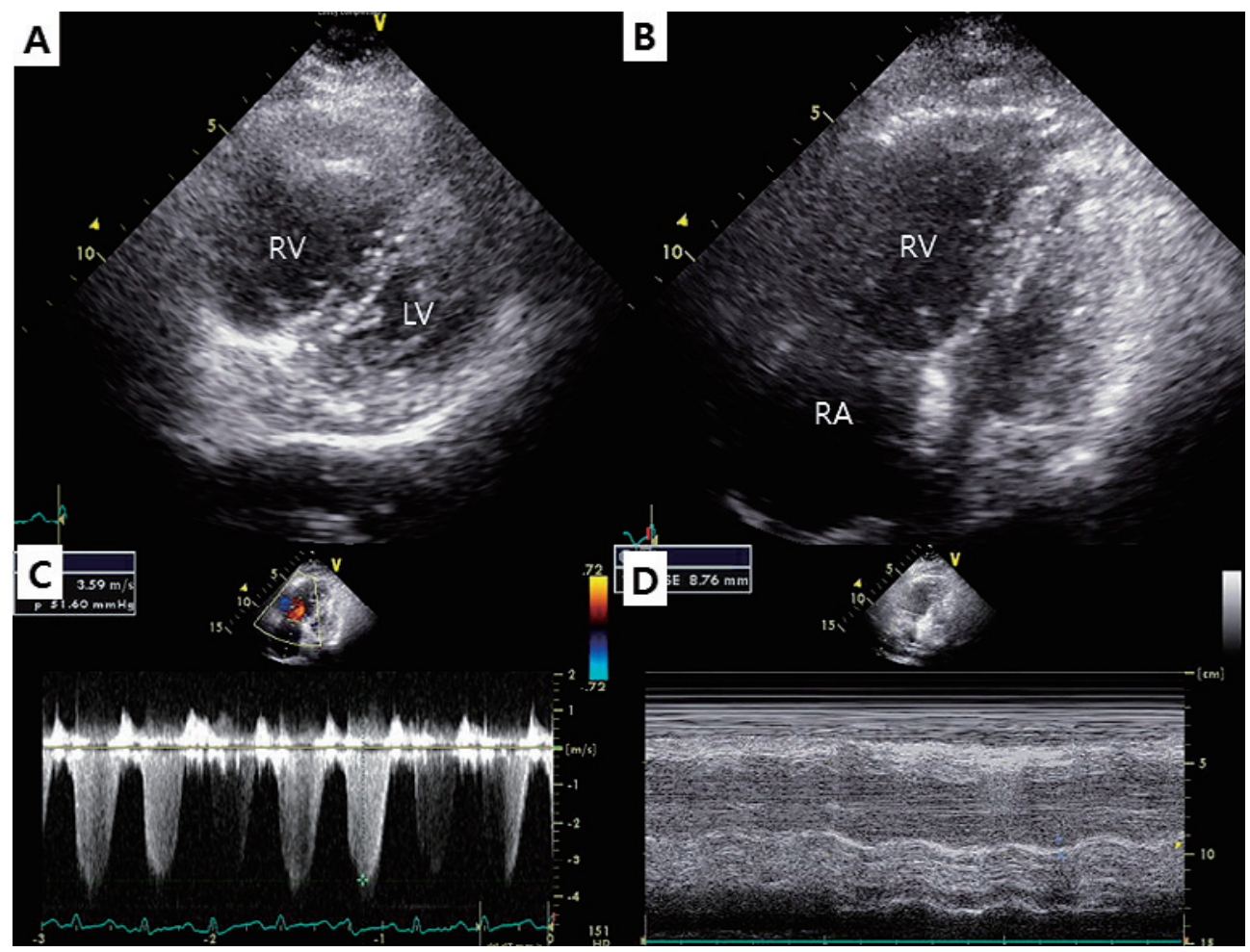

Figure 1. Transthoracic echocardiogram. Transthoracic echocardiography (TTE) revealed D-shaped left ventricle (A), dilated RA and RV cavities (B), pulmonary hypertension (C), and RV dysfunction (D).

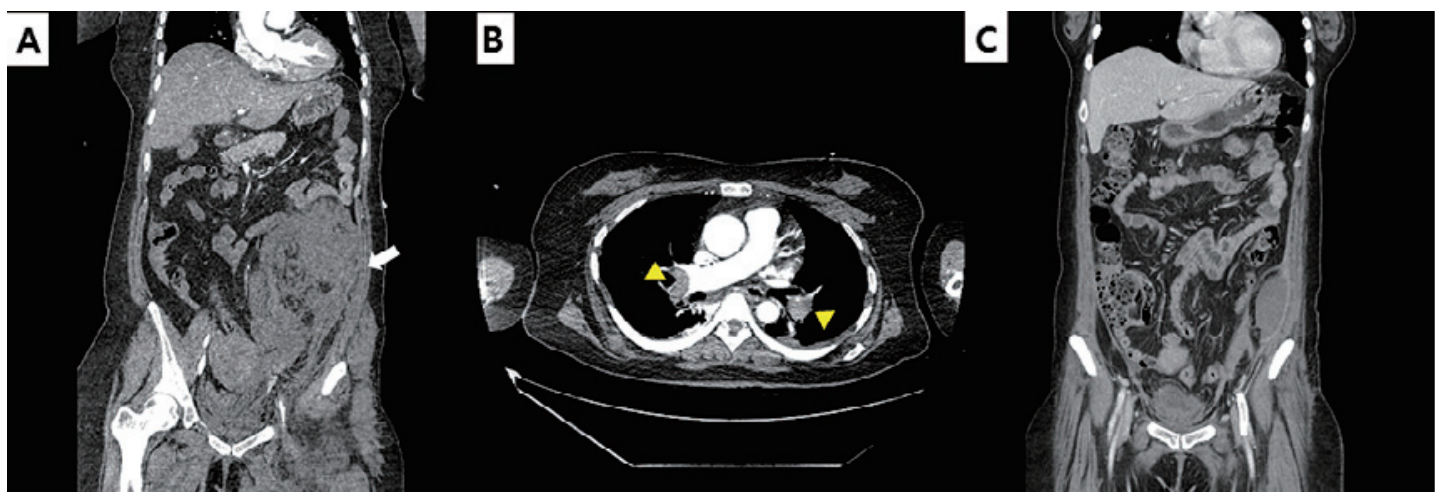

Figure 2. Computed tomography. Computed tomography findings show a massive hematoma (white arrow) along the left anterior and posterior pararenal space (A) and heavy thrombus (yellow arrowheads) in both pulmonary trunks (B). One month later, follow-up CT findings revealed regression of massive hematoma $(\mathbf{C})$.

kyo, Japan) emergent bypass system was used. The ECMO was successfully inserted by right femoral cannulation, but the patient was unstable due to active bleeding.

Computed tomography (CT) findings showed a massive hematoma along the left anterior and posterior pararenal space (Figure 2A) and thrombi in both pulmonary arteries (Figure 2B). Peripheral artery angiography was performed to manage vascular complications.

At first, a 5-Fr catheter was inserted in the left brachial artery using the cut-down procedure. Angiography showed perforation of the left CFA (Figure 3A). After occlusion of the perforation site using a $5 \times 40 \mathrm{~mm}$ balloon, the left inguinal area was opened using the cut-down pro- cedure to repair arterial bleeding. Unexpected venous injuries were also observed and were too severe to repair. The arterial bleeding site could not be approximated due to massive hematoma. Therefore, the $8 \mathrm{Fr}$ shuttle sheath (Cook Medical, Bloomington, USA) in the left brachial artery was replaced with an $8 \times 50 \mathrm{~mm}$ Viabahn stent graft (W.L. Gore, Flagstaff, Ariz) in the left CFA to occlude the bleeding site (Figure 3B). Fortunately, the patient was stable after stent placement. Unfractionated heparin was administered to reduce the PTE burden. The patient was decannulated six days after ECMO insertion. One month later, follow-up CT findings revealed regression of massive hematoma (Figure 2C) and the patient 


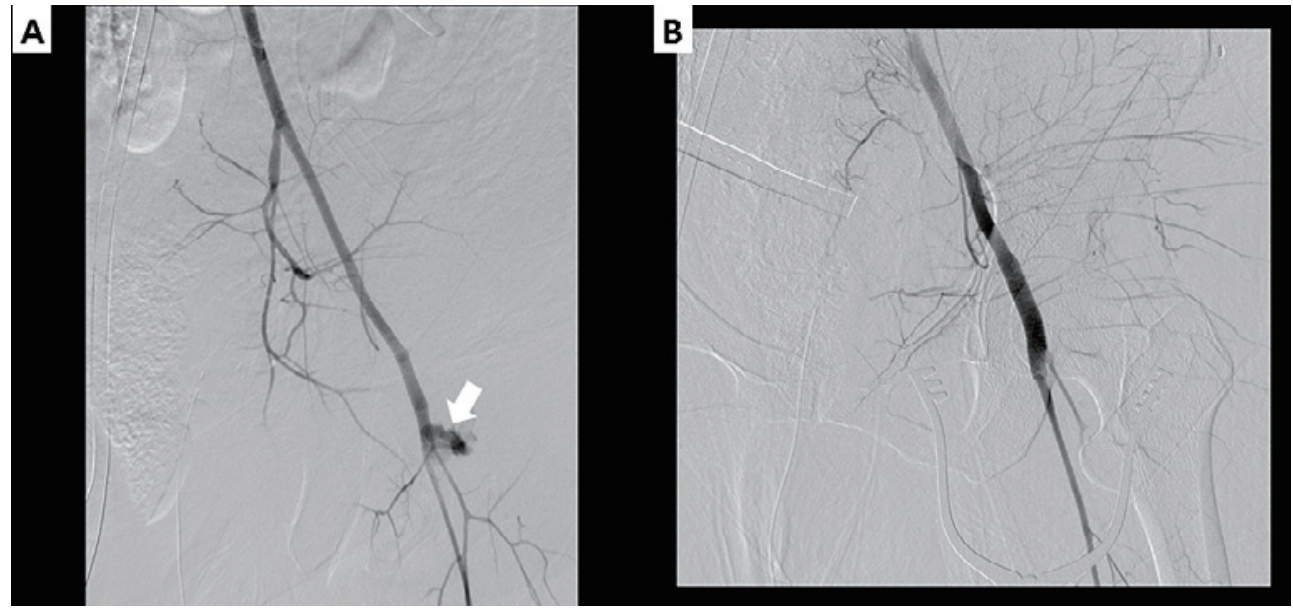

Figure 3. Peripheral angiography. Angiography showed perforation (white arrow) of left common femoral artery (CFA) (A). An $8 \times 50 \mathrm{~mm}$ Viabahn stent graft (W.L. Gore, Flagstaff, Ariz) was placed in the left CFA to occlude the bleeding site $(\mathbf{B})$.

was discharged without sequelae. The patient remains asymptomatic two years after discharge.

\section{Discussion}

In the present case, CFA perforation was encountered during cannulation for ECMO in a patient requiring CPR due to massive PE. The arterial bleed was successfully controlled using stent placement. To the best of our knowledge, there have been no reports of successful management of perforated CFA during cannulation for ECMO using a stent.

Massive PE is life-threatening. In the International Cooperative Pulmonary Embolism Registry (ICOPER) of 2454 consecutive patients from 7 countries, $88.9 \%$ were symptomatic and hemodynamically stable, and $4.2 \%$ were hemodynamically unstable. ${ }^{5}$ Thrombolysis is recommended as the standard treatment in patients with massive PE. $\left.{ }^{6}\right)$ However, major bleeding increases with thrombolysis, compared to heparin, and recurrent PE or death also occurred in some patients treated with thrombolysis. ${ }^{7}$ Other treatment options are open surgical embolectomy and catheter thrombectomy. However, pulmonary embolectomy results are not always satisfactory. If thrombolysis is selected as the first treatment, ECMO cannot be used to stabilize hemodynamic status because of the high risk of bleeding. Therefore, ECMO was inserted first in our case. V-A ECMO can provide lifesaving hemodynamic and respiratory support in critically ill patients with massive PE who require CPR or are too unstable to tolerate other treatment options. ${ }^{8,9}$

V-A ECMO decompresses the acutely overloaded right atrium and ventricle while stabilizing hemodynamic status in patients with massive PE. However, vascular complications occur in fewer than $20 \%$ of ECMO patients. Most patients require surgical intervention with a high technical success. ${ }^{4,10)}$ However, a surgical primary repair of arterial injuries may potentially increase cardiac damage in unstable patients. In addition, secondary complications after surgery, such as hematoma and postopera- tive infection, frequently develop. Successful endovascular management with covered stent has been proposed as an alternative to surgical repair. There are some benefits for endovascular management with covered stent, such as the procedure can be performed under local anesthesia, it can be completed rapidly, there is reduced morbidity associated with less invasive management, and patients with multiple comorbidities can be treated. ${ }^{11)}$ In our case, surgical intervention was difficult to perform due to the massive venous bleeding, and we chose endovascular management with covered stent.

Imaging modalities, including ultrasound and fluoroscopy, are used for cannulation of ECMO to prevent vascular complications. However, in our case, ECMO cannulation was performed without imaging modalities because this case was urgent. Vascular complications are associated with reduced survival. ${ }^{4,12}$ A previous report on vascular injury management stated that 18 of 101 patients undergoing ECMO via femoral cannulation developed peripheral vascular complications. Of these, 16 patients required surgical intervention and 8 patients required femoral endarterectomy with patch angioplasty. ${ }^{4}$ In our previous report, 19 of 95 patients developed cannula site bleeding in patients with cardiac failure requiring ECMO. One $(5.3 \%)$ was managed by using covered stent, two $(10.5 \%)$ were managed nonoperatively, and $16(84.2 \%)$ needed surgical intervention. Nine of the patients requiring surgery underwent primary repair and one underwent femoral endarterectomy with patch angioplasty. ${ }^{13)}$

ECMO's importance in CPR is expected to increase due to longer life expectancies. Interventional cardiologists are frequently challenged by vascular injury during cannulation for ECMO. Imaging modalities are recommended to prevent vascular complications during ECMO cannulation. However, if vascular injuries occur, stent placement should be considered for patients with arterial perforation during cannulation for ECMO. 


\section{Disclosures}

Conflicts of interest: None.

\section{References}

1. Maggio P, Hemmila M, Haft J, Bartlett R. Extracorporeal life support for massive pulmonary embolism. J Trauma 2007; 62: 570-6.

2. Kasper W, Konstantinides S, Geibel A, et al. Management strategies and determinants of outcome in acute major pulmonary embolism: results of a multicenter registry. J Am Coll Cardiol 1997; 30: 1165-71.

3. Buller HR, Agnelli G, Hull RD, Hyers TM, Prins MH, Raskob GE. Antithrombotic therapy for venous thromboembolic disease: the Seventh ACCP Conference on Antithrombotic and Thrombolytic Therapy. Chest 2004; 126: 401-28s.

4. Aziz F, Brehm CE, El-Banyosy A, Han DC, Atnip RG, Reed $\mathrm{AB}$. Arterial complications in patients undergoing extracorporeal membrane oxygenation via femoral cannulation. Ann Vasc Surg 2014; 28: 178-83.

5. Goldhaber SZ, Visani L, De Rosa M. Acute pulmonary embolism: clinical outcomes in the International Cooperative Pulmonary Embolism Registry (ICOPER). Lancet 1999; 353: 1386-9.

6. Kucher N, Goldhaber SZ. Management of massive pulmonary embolism. Circulation 2005; 112: e28-32.
7. Wan S, Quinlan DJ, Agnelli G, Eikelboom JW. Thrombolysis compared with heparin for the initial treatment of pulmonary embolism: a meta-analysis of the randomized controlled trials. Circulation 2004; 110: 744-9.

8. Chon MK, Park YH, Choi JH, et al. Thrombolytic therapy complemented by ECMO: successful treatment for a case of massive pulmonary thromboembolism with hemodynamic collapse. J Korean Med Sci 2014; 29: 735-8.

9. Watanabe Y, Sakakura K, Akashi N, et al. Veno-arterial extracorporeal membrane oxygenation with conventional anticoagulation can be a best solution for shock due to massive PE. Int Heart J 2017; 58: 831-4.

10. Kagawa E, Inoue I, Kawagoe T, et al. Assessment of outcomes and differences between in- and out-of-hospital cardiac arrest patients treated with cardiopulmonary resuscitation using extracorporeal life support. Resuscitation 2010; 81: 968-73.

11. Kufner S, Cassese S, Groha P, et al. Covered stents for endovascular repair of iatrogenic injuries of iliac and femoral arteries. Cardiovasc Revasc Med 2015; 16: 156-62.

12. Bisdas T, Beutel G, Warnecke G, et al. Vascular complications in patients undergoing femoral cannulation for extracorporeal membrane oxygenation support. Ann Thorac Surg 2011; 92: 626-31.

13. Lee SN, Jo MS, Yoo KD. Impact of age on extracorporeal membrane oxygenation survival of patients with cardiac failure. Clin Interv Aging 2017; 12: 1347-53. 